\title{
Valoración paisajística del potencial turístico de la reserva Río Playa de Comalcalco, Tabasco
}

\author{
Kristian Liévano Torres* \\ Universidad Popular de la Chontalpa \\ José Francisco Juárez López \\ Colegio de Postgraduados Campus Tabasco \\ María Lyssette Mazo Quevedo \\ Universidad Popular de la Chontalpa
}

\section{Resumen}

Con base en la percepción y valoración paisajística, el presente trabajo tiene por objetivo identificar el potencial turístico de la reserva ecológica Río Playa, ubicada en el municipio de Comalcalco, Tabasco. Se realizó un inventario de los recursos paisajísticos de la zona de estudio y se analizaron los factores sociales y económicos de comunidades aledañas, eligiendo aquellas donde habitan los ejidatarios a cargo de la administración de la reserva, pues dentro de esta no existe una población representativa que permita llevar a cabo el análisis. Posteriormente se determinaron seis unidades de paisaje a través de mapas temáticos, tomas fotográficas en campo, marco referencial y opiniones de especialistas. Estas unidades fueron evaluadas por dos grupos de expertos: alumnos y docentes de distintas áreas de conocimiento, como turismo, agronomía y biología, todos seleccionados de manera aleatoria. Esta evaluación permitió conocer el potencial turístico de cada unidad paisajística. Se concluyó que, por sus características paisajísticas, la reserva ecológica Río Playa cuenta con potencial para practicar actividades de turismo de naturaleza, sobre todo en el segmento de ecoturismo. Por lo tanto, esta investigación tuvo un aporte descriptivo que permitió conocer la percepción de expertos sobre los paisajes de la reserva y proponer actividades turísticas dentro de un marco de sustentabilidad.

\section{Palabras clave}

Valoración paisajística, recursos naturales, potencialidad turística.

*Correo electrónico: kristy_cta@hotmail.com, juarezlo@colpos.mx, marialyssete@hotmail.com

Recibido: 30/03/2014 · Aceptado: 25/04/2014




\title{
Tourism potential of the Río Playa Reserve in Comalcalco, Tabasco based on the landscape value
}

\author{
Kristian Liévano Torres* \\ Universidad Popular de la Chontalpa \\ José Francisco Juárez López \\ Colegio de Postgraduados Campus Tabasco \\ María Lyssette Mazo Quevedo \\ Universidad Popular de la Chontalpa
}

\begin{abstract}
The present research work identifies the tourism potential of the ecological reserve based on the tourist's perception and landscape value. An inventory of the existing landscape resources was made and economic and social factors of nearby communities were analyzed. Communities where "ejidatarios" are managers of the natural reserve were selected since population within the reserve is limited and does not allow the analysis. Six landscape units were identified by thematic maps, photographs were taken in situ, a referential framework was established and expert opinions by two randomly selected expert groups, students and professors from different knowledge areas, such as tourism, agriculture and biology, evaluated these units. As a conclusion it was established that, through a descriptive process and based on the landscape characteristics, the Ecological Reserve of Río Playa has the potential of supporting nature tourism activities mainly in the ecotourism field within the framework of sustainability.
\end{abstract}

\section{KEY WORDS}

Landscape value, natural resources, tourism potential

*E-mail: larroyo@uqroo.edu.mx, lopuslopez@yahoo, romanogino@hotmail.com 


\section{Introducción}

El turismo está creciendo en todo el mundo y México no es la excepción. La Comisión Nacional de Áreas Naturales Protegidas (Conanp) estima que alrededor de 5.5 millones de turistas visitan cada año las áreas protegidas federales (APF), y estudios de la Secretaría de Turismo (Sectur) muestran que estas áreas son los destinos favoritos para desarrollar actividades de turismo de naturaleza (Conanp-Semarnat, 2007).

El estado de Tabasco cuenta con 13 áreas naturales protegidas (ANP), dos de las cuales son de control federal y 11 de control estatal. De las primeras, una es reserva de la biósfera y otra área de protección de flora y fauna; de las segundas, seis son reservas ecológicas, dos parques estatales, dos parques ecológicos y una monumento natural. La reserva ecológica Río Playa, zona de interés para la presente investigación, es de control estatal y fue decretada como ANP el 29 de septiembre de 2004 (Sedespa, 2006).

La inquietud por identificar el potencial turístico de esta zona se debe a que alberga una gran diversidad biológica. En este sentido, el trabajo se concreta en una evaluación paisajística de la reserva ecológica Río Playa para conocer la percepción de expertos sobre las características de sus paisajes, así como en el desarrollo de propuestas de actividades especializadas en el segmento de turismo de naturaleza.

\section{Objetivo}

El objetivo general de esta investigación es establecer el potencial turístico de la reserva ecológica Río Playa mediante la evaluación visual del paisaje. Los objetivos específicos son:

- Realizar un inventario de los recursos paisajísticos con los que cuenta la reserva.

- Analizar de forma generalizada los factores sociales y económicos de las comunidades aledañas a la zona de estudio: Zapotal $2^{a}$ sección e Ignacio Zaragoza $4^{\text {a }}$ sección, del municipio de Comalcalco, y Los Potreritos, del municipio de Paraíso. 
- Identificar el potencial de la reserva con base en un estudio de percepción y valoración de la calidad visual del paisaje.

- Ubicar las actividades propias de turismo de naturaleza que pueden llevarse a cabo en la zona, de acuerdo con el potencial turístico definido por expertos en el área.

\section{Antecedentes}

La Procuraduría Federal de Protección al Ambiente (Profepa) define las ANP como zonas del territorio nacional donde los ambientes originales no han sido significativamente alterados por la actividad del hombre o que requieren ser preservados o restaurados. Estas se establecen mediante decreto presidencial y las actividades que pueden llevarse a cabo en ellas se determinan de acuerdo con la Ley General del Equilibrio Ecológico y la Protección al Ambiente (LGEEPA) y su reglamento, así como con los programas de conservación y manejo y de ordenamiento ecológico (Profepa-Semarnat, 2012).

La visita de turistas a las Anp representa un indicador para el desarrollo de propuestas de turismo en estas áreas del territorio. El turismo de naturaleza en cualquiera de sus segmentos (ecoturismo, de aventura y rural) se presenta como una oportunidad para el aprovechamiento de los recursos (Cestur-Sectur y UAM, 2007).

En referencia a los espacios poco intervenidos, donde la actividad humana no ha alterado significativamente el ambiente, Pires (2011) menciona que "el paisaje predominante natural atrae por la presencia y composición escénica de los elementos naturales como el agua, la vegetación, la geografía, y la fauna, además de la estacionalidad y los episodios climáticos” (pág. 523).

Los estudios sobre el paisaje percibido provienen de diversas disciplinas. Para Muñoz-Pedreros (2004), el paisaje puede estudiarse desde dos direcciones: desde un sentido estructural, a través del método indirecto, y desde un sentido visual, por medio de un método directo.

Para los fines de esta investigación, el paisaje se abordará desde una perspectiva visual asociada a sensaciones y recuerdos, por lo que depende de muchas condiciones internas del observador (Aramburu et al., 2005). Este método 
valora la unidad de paisaje (UP) mediante la contemplación total directamente en la zona o a través de fotografías y diapositivas, en el entendido de que las up son espacios homogéneos de terreno. Priego Santander et al. (2008) la definen como la mínima unidad cartografiable que permite representar los principales componentes de un ecosistema.

El estudio fue realizado en la reserva ecológica ejidal campesina Río Playa, ubicada en la subregión de la Chontalpa tabasqueña, en el municipio de Comalcalco. El ejido Río Playa se localiza entre las coordenadas $18^{\circ} 19^{\prime} 00^{\prime \prime}$ y $18^{\circ}$ $22^{\prime} 00^{\prime \prime}$ de latitud norte y entre los $93^{\circ} 17^{\prime} 00^{\prime \prime}$ y $93^{\circ} 18^{\prime} 00^{\prime \prime}$ de longitud oeste. Tiene una superficie total de 1137.61 hectáreas (INEGI, 2010a).

La reserva colinda con la comunidad de Potreritos, perteneciente al municipio de Paraíso, se ubica dentro de la llanura costera del Golfo Sur y se caracteriza por tener una pendiente menor al dos por ciento, lo cual favorece la acumulación de agua superficial. La figura 1 muestra, además de la zona de estudio, tres comunidades que se tomaron como referencia en el análisis socioeconómico por las razones que se expondrán a lo largo del trabajo. Estas comunidades son I. Zaragoza $4^{\text {a }}$ sección y Zapotal 2a sección, de Comalcalco, y Potreritos, de Paraíso.

Río Playa es la primera reserva estatal promovida para su decreto por un grupo de 44 ejidatarios que conforman la Sociedad de Solidaridad Social (sss) denominada Los pochitoques. Desde que se emitió el decreto, se ha manejado y administrado como reserva ecológica. En 2003, los agricultores de esta organización recibieron el Premio Estatal de Ecología por su contribución a la conservación de su ejido (López-Hernández y Rodríguez Luna, 2007). 




Fuente: Elaboración propia con base en la carta topográfica de Comalcalco (E15A79), escala 1:50 000 (INEGI, 2000).

Figura 1. Localización del área de estudio: Río Playa y comunidades aledañas (I. Zaragoza $4^{a}$ sección, Zapotal $2^{a}$ sección y Potreritos) 


\section{Metodología}

Este trabajo es un estudio cualitativo basado en la interpretación. Se trata de una investigación aplicada que busca la solución de un problema concreto y tiene una vinculación muy estrecha con la realidad social, por lo que al interpretar y valorar los resultados tiene repercusiones en una serie de condicionantes.

La primera parte (coincidente con el primer objetivo) consistió en el levantamiento del inventario; se efectuó una revisión de literatura para tener referencias de las especies de flora y fauna que habitan o habitaron en el sitio. La principal fuente de información fue una cédula de las ANp de Tabasco publicada por la Secretaría de Desarrollo Social y Protección al Ambiente (Sedespa, 2006), además de publicaciones sobre estudios y proyectos que la Universidad Juárez Autónoma de Tabasco (UJAT) ha llevado a cabo en la región. En esta etapa se realizó un primer recorrido para identificar y posteriormente evaluar los recursos naturales con los que cuenta la zona. Durante el recorrido se tomaron 67 fotografías desde diferentes ángulos, usando una cámara convencional de 16.1 megapixeles, luego se seleccionaron cinco fotografías y se describieron las características básicas comunes de cada recurso, para lo que se utilizó una ficha propuesta por la Sectur (2000) en su fascículo Planeación y gestión del desarrollo turístico municipal.

Los recursos fueron jerarquizados mediante una valoración del 0 al 5, propuesta por Cárdenas Tabares (2006), donde 0 corresponde a atractivos con cualidades que no permiten incorporarlos a la jerarquía 1, que es el umbral mínimo de jerarquización y que corresponde a atractivos sin méritos, cuyos atributos solo son conocidos a nivel local y no implican pernocte; en la jerarquía 2 se encuentran atractivos con un radio de influencia mayor pero no lo suficiente para motivar por sí solos el viaje turístico; la jerarquía 3 corresponde a atractivos con algún rasgo llamativo con la posibilidad de interesar a visitantes del mercado interno o externo que hubiesen llegado a la zona por otros intereses turísticos; la jerarquía 4 representa atractivos con rasgos excepcionales en un país, capaces de motivar una corriente de visitantes del mercado interno y externo, pero en menor porcentaje que la jerarquía 5, ya que esta última corresponde a un atractivo excepcional de gran significación para el mercado turístico internacional, con el potencial de motivar por sí solo una corriente de visitantes que 
represente un porcentaje considerable del total de la demanda del país. De esta manera se le dio un valor a cada recurso identificado en el área de estudio.

Para cumplir con el segundo objetivo se seleccionaron dos fuentes de información: en primer lugar se utilizaron datos de Tabasco generados por el Instituto Nacional de Estadística y Geografía (INEGI, 2010a). Los principales indicadores socioeconómicos evaluados a través de esta fuente fueron: tamaño de población, migración, indigenismo, grado de escolaridad, servicios de salud, población económicamente activa, inactiva y ocupada; total de viviendas y características de las mismas. Cabe señalar que, por cuestiones de confiabilidad, con los censos del INEGI no es posible generar datos para unidades geográficas con menos de tres viviendas, por lo que para localidades como Río Playa solo se muestra la población total, las viviendas totales y el total de viviendas habitadas. Por esta razón, el análisis se basa en las comunidades a las que pertenecen los ejidatarios a cargo del área, que en todo caso serían los beneficiados directos de cualquier proyecto en la zona. Las comunidades seleccionadas fueron, como ya se dijo: I. Zaragoza $4^{a}$ sección y Zapotal $2^{\text {a }}$ sección, de Comalcalco, y Potreritos, de Paraíso.

Como segunda fuente de información se recurrió a los integrantes de la mesa directiva de los ejidatarios a cargo de la reserva: el comisariado ejidal, José de la Luz Domínguez González; el secretario, Rutilo Hernández León, y el tesorero, Calletano Castellano Castellano, a quienes se entrevistó. Esto permitió conocer a fondo las condiciones actuales del manejo de la reserva, los proyectos desarrollados y su interés por emprender otros nuevos, así como la principal actividad económica en la que se desenvuelven. Además, se habló con el señor Virgilio González Lázaro, quien fungió como comisariado ejidal cuando Río Playa fue decretado Anp en la categoría de reserva ecológica, así como con el doctor Gabriel Márquez Coturier, quien desarrolló diferentes proyectos en la zona durante el periodo de comisariado citado.

El tercer objetivo se llevó a cabo en tres etapas: primero se identificaron las up, para esto se hizo una revisión de la literatura disponible sobre la flora y fauna predominante en la zona, después se hizo un análisis cartográfico de gabinete, donde mediante mapas temáticos se establecieron seis up, considerando la cubierta dominante, los cuerpos de agua presentes y la intervención humana 
observable (Aramburu et al., 2005). Estos mapas fueron generados en el laboratorio de geomática del Colegio de Postgraduados Campus Tabasco, utilizando el sistema de información geográfica ArcGIS 10.0 y con base en las áreas geoestadísticas municipales del INEGi (2010b) a una escala de 1:150 000. Por último, se realizó un segundo recorrido por la zona en el cual, con la misma cámara que se usó en el primer recorrido, se tomaron 50 fotografías de las unidades paisajísticas identificadas. Esto permitió corroborar la información obtenida en la revisión del marco referencial y los mapas temáticos. Más adelante, con la ayuda de expertos y con las fotografías, se describieron las características básicas de cada unidad de paisaje.

Para evaluar las up y su uso turístico se aplicó una encuesta grupal a expertos en las áreas de biología, turismo y agronomía. La evaluación se efectuó en dos grupos: uno de alumnos y otro de docentes, ambos de la Universidad Popular de la Chontalpa (UPCH). En el primer grupo se seleccionaron alumnos de las licenciaturas en Agronomía y en Turismo Alternativo de segundo a octavo semestre, utilizando una muestra aleatoria no probabilística, donde se eligió al azar el diez por ciento de cada grupo; cabe señalar que solo se tuvieron en cuenta los grupos disponibles al momento del sorteo. Con el fin de que la mayor parte de los estudiantes estuviese presente, y debido a que estas carreras se imparten en el turno matutino, el sorteo se realizó de 10:30 a 11:00 am, lo que dio un total de diez alumnos, siete de la carrera de turismo y tres de agronomía.

Para el segundo grupo de evaluadores, correspondiente a docentes, se consideraron expertos en turismo de naturaleza, biólogos y agrónomos provenientes de la Academia de Turismo Alternativo y Agronomía. Se tomó una muestra de seis evaluadores, tres de los cuales son especialistas en turismo de naturaleza, dos biólogos y uno agrónomo. En ambos grupos se aplicó el mismo cuestionario y se dejaron dos preguntas abiertas para que el evaluador expresara libremente su opinión sobre determinados criterios que pudieran haberse pasado por alto en la elaboración del cuestionario.

La evaluación paisajística se basa en la metodología propuesta por MuñozPedreros (2004) a través del método directo; así, la valoración se realiza a partir de la contemplación total del paisaje. Se preparó una presentación con diapositivas para que los expertos, apoyados en un cuestionario, valoraran las 146 fotografías con un tiempo de 15 minutos para responder las preguntas y dar una calificación sobre el atractivo. 
El cuestionario se fundamentó en dos metodologías: a) de un estudio de cartografía del paisaje llevado a cabo en La Rioja, España. Utilizando una escala que evalúa el paisaje en función de cuánto gusta (incluye las categorías: muy poco, poco, algo, bastante y mucho) y que permite evaluar las características más llamativas del paisaje (Aramburu et al., 2005). La segunda es una lista de adjetivos propuesta por Muñoz-Pedreros (2004), que considera las categorías: feo, sin interés, agradable, distinguido, fantástico y espectacular; cada categoría está compuesta por ciertos adjetivos que los evaluadores seleccionaron de acuerdo con su percepción. Posteriormente se preguntó a los encuestados qué uso turístico le darían a los recursos dentro de los paisajes, lo que permitió determinar su potencial y, al mismo tiempo, cumplir el último objetivo de la investigación.

\section{Resultados y discusión}

Hace aproximadamente diez años, en Río Playa abundaban especies nativas como pejelagartos, quelonios, mojarras, pijijes y demás fauna característica de la región tropical (cuadro 1). Sin embargo, la apertura de un canal hacia el mar en el vecino municipio de Paraíso cambió la salinidad del agua y, por ende, el ecosistema del lugar.

El ex comisariado Virgilio González Lázaro indicó que la vegetación comenzó a cambiar con la siembra de manglares y especies acuáticas como jaibas, caracoles y otros crustáceos. ${ }^{1}$ Con todo, durante el recorrido por la zona se pudieron observar algunos ejemplares de pelícanos, patos, gaviotas y otras aves que permanecen durante la mayor parte del año.

Dentro de la reserva se identificaron cinco recursos paisajísticos naturales: vegetación hidrófita, compuesta principalmente por popal y tular; cuerpos de agua pertenecientes al río Aguas Negras, el cual se comunica mediante un canal expandido por Petróleos Mexicanos (Pemex) con la laguna Las Flores, que forma parte del complejo que desemboca al mar en la Barra de Tupilco; también se hallaron áreas pequeñas de tinto, pastizal cultivado e inundable y, por último, manglar, en su mayoría mangle blanco (Laguncularia racemosa) (cuadro 2).

${ }^{1}$ Entrevista personal, 18 de septiembre de 2013. 


\section{Cuadro 1. Últimos inventarios levantados en la reserva}

\begin{tabular}{|c|c|}
\hline \multicolumn{2}{|r|}{ VEGETACIÓN } \\
\hline Córdoba (2001) & $\begin{array}{l}\text { Estima } 166 \text { especies de plantas: } 89 \text { herbáceas, } 29 \text { arbóreas, } 17 \text { enredaderas, } \\
12 \text { arbustos, diez epifitas, cuatro palmas, cuatro géneros de bejuco y una } \\
\text { parásita. }\end{array}$ \\
\hline Gómez (1999) & $\begin{array}{l}\text { Encontró que la vegetación correspondía a humedales con una comunidad } \\
\text { dominante de popal (Thalia geniculata) asociada con especies como moli- } \\
\text { nillo (Cyperus giganteus), chintul (Cyperus articulatus) y pastos manatinero } \\
\text { (Hymenachne amplexicaulis) y lamedor (Leersia hexandra). Otros tipos de } \\
\text { pastizales compuestos por pelusa (Echinochloa polystachya), arrocillo (Echi- } \\
\text { nochloa colonum), camalote (Echinochloa crus-galli) y egipto (Panicum pur- } \\
\text { purascens). También localizó tres manchones de selva con especies arbóreas } \\
\text { como palo fierro (Ocotea cernua), ceiba (Ceiba petandra), macuili (Tabebuia } \\
\text { rosea), zapote de agua (Pachira aquatica), jahuacte (Bactris balanoidea), } \\
\text { manchones de tinto (Haematoxylum campechianum) y un área de produc- } \\
\text { ción agrícola de temporal. }\end{array}$ \\
\hline \multicolumn{2}{|r|}{ FAUNA } \\
\hline Sánchez (2001) & $\begin{array}{l}\text { Asume la presencia de } 80 \text { especies constituidas por una diversidad de tres } \\
\text { anfibios, ocho reptiles, } 52 \text { aves y } 17 \text { mamíferos en lo referente a especies } \\
\text { terrestres. De la fauna acuática registró diez familias con } 13 \text { géneros y } 19 \text { es- } \\
\text { pecies en el dren de cinco kilómetros que desemboca en el río Aguas Negras } \\
\text { y que tiene comunicación con la laguna Tupilco. Algunas especies represen- } \\
\text { tativas en la reserva son: hicotea (Trachemys scripta), mazacúa (Boa cons- } \\
\text { trictor), iguana (Iguana iguana), aspoque (Ctenosaura similis), guío (Buteo } \\
\text { magnirostris), halcón (Falco femoralis), zarzal (Glaucidium brasilianum), } \\
\text { chipe charquero (Seiurus noveboracensis). }\end{array}$ \\
\hline $\begin{array}{l}\text { Especies que } \\
\text { requieren } \\
\text { protección } \\
\text { especial Nom-059- } \\
\text { ECOL-2001 }\end{array}$ & $\begin{array}{l}\text { Caracolero (Rostrhamus sociabilis), perico (Aratinga nana astec), chiqui- } \\
\text { guao (Chelydra serpentina), pochitoque (Kinosternon leucostomum), guao } \\
\text { (Staurotypus triporcatus) y el pez fil (Rhamdia guatemalensis). De las espe- } \\
\text { cies amenazadas que se encontraron pueden mencionarse el perro de agua o } \\
\text { nutria (Lutra longicaudis), el halcón fajado (Falco femoralis) y el reptil sau- } \\
\text { yan o mazacúa (Boa constrictor). La única especie en peligro de extinción } \\
\text { que se registró es el saraguato (Alouatta palliata). }\end{array}$ \\
\hline
\end{tabular}

Fuente: Elaboración propia con base en López-Hernández y Rodríguez Luna (2007) y la cédula de las ANp de Tabasco (Sedespa, 2006). 
Cuadro 2. Evaluación y jerarquización de los recursos paisajísticos naturales

\begin{tabular}{|c|c|}
\hline VEGETACIÓN HIDRÓFITA & Río AGUAS NEGRAS \\
\hline Jerarquía: 1 & Jerarquía: 2 \\
\hline Categoría: recurso natural & Categoría: recurso natural \\
\hline Uso de suelo: área protegida & Uso de suelo: área protegida \\
\hline Accesibilidad: fácil & Accesibilidad: fácil \\
\hline Estado de conservación: bueno & Estado de conservación: malo \\
\hline Calidad de entorno: alta & Calidad de entorno: alta \\
\hline Evaluación general: bueno & Evaluación general: bueno \\
\hline Actividad relacionada: turismo & $\begin{array}{l}\text { Actividad relacionada: turismo } \\
\text { y pesca }\end{array}$ \\
\hline Pastizal & MANGLAR \\
\hline Jerarquía: 1 & Jerarquía: 3 \\
\hline Categoría: recurso natural & Categoría: recurso natural \\
\hline Uso de suelo: área protegida & Uso de suelo: área protegida \\
\hline Accesibilidad: fácil & Accesibilidad: fácil \\
\hline Estado de conservación: bueno & Estado de conservación: bueno \\
\hline Calidad de entorno: alta & Calidad de entorno: alta \\
\hline Evaluación general: bueno & Evaluación general: bueno \\
\hline $\begin{array}{l}\text { Actividad relacionada: turismo, } \\
\text { agricultura y ganadería }\end{array}$ & Actividad relacionada: turismo \\
\hline
\end{tabular}

Fuente: Elaboración propia con base en la ficha de inventario de la Sectur (2000).

A Río Playa se puede llegar fácilmente, pues la carretera se encuentra en buenas condiciones y dispone de servicio de transportes cada 30 minutos. Dentro de la reserva solo es posible trasladarse de un recurso a otro por medio de senderos o en lancha. Cabe mencionar que estos paisajes no han sido fuertemente intervenidos por el desarrollo urbano, por lo que se percibe un entorno armonioso y agradable.

La infraestructura con la que cuenta la reserva es de diez piletas, dos bodegas, lanchas, un puente de madera y cercas de alambre que dividen los terrenos. También existen algunos senderos formados por el paso de los pobladores. Dentro de la reserva no hay señalizaciones y su nivel de afluencia actual es casi nulo. Con anterioridad se realizaron proyectos que atraían turismo proveniente de universidades, cuya finalidad era llevar a cabo investigaciones biológicas, pero ahora ya no se trabaja en esos proyectos.

Las principales actividades económicas de los ejidatarios son la agricultura (en particular la siembra de maíz y frijol) y la pesca de tilapia, ambos para 
Cuadro 3. Datos socioeconómicos de las comunidades seleccionadas para su análisis

\begin{tabular}{|c|c|c|c|}
\hline INDICADOR & $\begin{array}{l}\text { ZAPOTAL } 2^{a} \\
\text { SECCIÓN }\end{array}$ & $\begin{array}{l}\text { I. ZARAGOZA } 4^{\text {a }} \\
\text { SECCIÓN }\end{array}$ & POTRERITOS \\
\hline Población total & 1952 & 349 & 1212 \\
\hline Población nacida en la entidad & 1935 & 349 & 1195 \\
\hline $\begin{array}{l}\text { Población mayor de cinco años } \\
\text { residente en otra entidad }\end{array}$ & 8 & 0 & 6 \\
\hline $\begin{array}{l}\text { Población mayor a tres años } \\
\text { hablante de lengua indígena }\end{array}$ & 1 & 0 & 2 \\
\hline Población de 15 años y más analfabeta & 141 & 17 & 65 \\
\hline $\begin{array}{l}\text { Población de } 15 \text { años y más sin } \\
\text { escolaridad }\end{array}$ & 73 & 13 & 28 \\
\hline $\begin{array}{l}\text { Población mayor de edad con } \\
\text { educación posbásica }\end{array}$ & 142 & 16 & 92 \\
\hline Población económicamente activa & 639 & 100 & 432 \\
\hline Población ocupada & 620 & 100 & 425 \\
\hline $\begin{array}{l}\text { Población derechohabiente de } \\
\text { servicios de salud }\end{array}$ & 1309 & 182 & 1012 \\
\hline Total de viviendas habitadas & 408 & 76 & 258 \\
\hline Viviendas con piso de material & 355 & 73 & 229 \\
\hline Viviendas con piso de tierra & 53 & 3 & 29 \\
\hline Viviendas con un dormitorio & 137 & 22 & 121 \\
\hline $\begin{array}{l}\text { Viviendas que disponen de } \\
\text { luz eléctrica }\end{array}$ & 404 & 76 & 254 \\
\hline $\begin{array}{l}\text { Viviendas que disponen } \\
\text { de agua entubada }\end{array}$ & 354 & 75 & 212 \\
\hline $\begin{array}{l}\text { Viviendas que disponen } \\
\text { de excusado o sanitario }\end{array}$ & 401 & 76 & 220 \\
\hline Viviendas que disponen de drenaje & 403 & 76 & 219 \\
\hline $\begin{array}{l}\text { Viviendas que no disponen de ningún } \\
\text { bien (radio, tv, internet, refrigerador, } \\
\text { lavadora, automóvil, teléfono fijo, } \\
\text { celular, etcétera) }\end{array}$ & 14 & 3 & 11 \\
\hline
\end{tabular}

150 Fuente: Elaboración propia con base en datos del INEGI (2010a). 
consumo propio. La mesa directiva de ejidatarios está interesada en impulsar actividades de turismo de naturaleza como una alternativa de desarrollo sustentable para sus comunidades. Debe decirse que pocos pobladores tienen conocimiento de la reserva, incluso algunos ejidatarios desconocían que fuese un ANP.

Los lugareños aún conservan muchos conocimientos adquiridos en capacitaciones anteriores, los cuales corresponden en su mayoría a la producción de especies como pejelagarto, venado, tortugas, así como a la reforestación de mangle y otros, lo cual representa una fortaleza para proyectos futuros.

Además, en las localidades seleccionadas para el análisis socioeconómico se percibe un estado social y económico característico de comunidades rurales (cuadro 3). La población cuenta con una carretera principal pavimentada, aunque algunas calles aún no lo están; pese a ello, el servicio de transporte público es constante. Hay un jardín de niños, escuelas primarias, secundarias y bachillerato, además de servicio médico, y algunos hogares tienen acceso al servicio de televisión de paga.

Para el manejo sostenible de la reserva, en 2001 se creó una unidad de manejo para la conservación de la vida silvestre (UMA) de venado cola blanca (Odocoileus virginianus) y diferentes especies de tortuga, como la tortuga blanca (Dermatemys mawii), guao o tres lomos (Staurotypus triporcatus), hicotea (Trachemys scripta), pochitoque (Kinosternon leucostomum), mojina (Rhinoclemmys areolata), chiquiguao (Chelydra serpentina) y taiman o joloca (Claudius angustatus), así como un centro reproductor de pejelagarto (Sedespa, 2006). No obstante, nada de lo anterior existe hoy en día, ya que los conflictos entre ejidatarios provocaron su abandono total. En la actualidad se trabaja en un proyecto de reforestación de mangle donde solo participan 33 ejidatarios, quienes manifestaron la necesidad de asesoría por parte de expertos, sobre todo en el ramo turístico, para la creación de proyectos en la zona.

Las seis up que se identificaron en la zona de estudio son: vegetación hidrófita (UP1), cuerpos de agua (up2), tintal (up3), pastizal (up4), manglar (up5) y asentamiento humano o poblados (UP6) (figura 2). 




Nota: Los cacaotales no pertenecen a la zona evaluada.

Fuente: Elaboración propia con base en la carta topográfica de Comalcalco (E15A79), escala 1:50 000 (INEGI, 2000).

Figura 2. Mapa de unidades de paisaje en la reserva 
Las up que obtuvieron categorías más altas y en las que ambos grupos estuvieron de acuerdo fueron la 2 y la 5 . Los factores que determinaron esta elección fueron el tipo de vegetación, la presencia de agua, la naturalidad y el fondo escénico. Este último principalmente en la up2 (cuadro 4).

Respecto al potencial identificado para cada unidad paisajística, la uP1 fue señalada en su mayoría para conservación por ambos grupos de encuestados. Cabe señalar que esta unidad es la mejor conservada y la que alberga una población más grande de especies de flora y fauna. En el cuadro 4 se muestran las actividades de ecoturismo que consideraron aquellos encuestados que optaron por darle un uso turístico.

La up2 y la up5 alcanzaron la categoría más alta en la evaluación de los alumnos: los principales usos señalados para la primera fueron turístico y de pesca (una combinación de ambos sería factible). Otra unidad elegida para dos tipos de uso fue la 3, considerada sobre todo para conservación, sin embargo, quienes optaron por un uso turístico mencionaron principalmente actividades del segmento de ecoturismo.

La up4 fue seleccionada en su mayoría para uso agrícola, ganadero y turístico; de la combinación de estas actividades surgen el turismo rural y el ecoturismo, por lo que algunos encuestados propusieron actividades que corresponden a estos segmentos del turismo de naturaleza.

La up5 obtuvo la categoría más alta por ambos grupos de evaluadores; los resultados arrojaron uso de conservación y turístico, lo cual es un buen indicador para los ejidatarios, pues su intención con la reforestación de mangle es iniciar proyectos de ecoturismo.

Por último, la up6 obtuvo la valoración más baja; en esta solo existe una casa habitada y otras construcciones que corresponden a bodegas y casas abandonadas, las cuales, considerando el potencial que alcanzó la reserva, podrían habilitarse para brindar servicios de hospedaje, restaurantes que ofrezcan gastronomía local o módulos de información. Esta unidad se encuentra a la entrada de la reserva. 
Cuadro 4. Descripción y valoración de la calidad visual de las Unidades Paisajísticas

\begin{tabular}{|c|c|c|c|}
\hline UNIDAD PAISAJÍSTICA & Resultados & ACTIVIDAD TURISTICA & FOTOGRAFÍA \\
\hline $\begin{array}{l}\text { UP1 vegetación } \\
\text { Hidrófita }\end{array}$ & $\begin{array}{l}\text { Docentes } \\
\text { *Me gusta algo } \\
\text { *Categoría: agradable } \\
\text { Alumnos } \\
\text { *Me gusta bastante/ } \\
\text { mucho } \\
\text { *Categoría: agradable }\end{array}$ & $\begin{array}{l}\text { Ecoturismo } \\
\text { * Observación y rescate } \\
\text { de flora y fauna } \\
\text { *Investigación biológica } \\
\text { *Safari fotográfico }\end{array}$ & \\
\hline $\begin{array}{l}\text { UP2 } \\
\text { Cuerpos de agua }\end{array}$ & $\begin{array}{l}\text { Docentes } \\
\text { *Me gusta bastante } \\
{ }^{*} \text { Categoría: distinguido } \\
\text { Alumnos } \\
\text { *Me gusta bastante } \\
\text { * Categoría: fantástico }\end{array}$ & $\begin{array}{l}\text { Ecoturismo } \\
\text { * Observación de eco- } \\
\text { sistema } \\
\text { *Investigación biológica } \\
\text { *Paseo en lancha } \\
\text { * Kayaquismo } \\
\text { *Pesca recreativa }\end{array}$ & \\
\hline $\begin{array}{l}\text { UP3 } \\
\text { Tintal }\end{array}$ & $\begin{array}{l}\text { Docentes } \\
\text { *Me gusta mucho } \\
\text { *Categoría: agradable } \\
\text { Alumnos } \\
\text { *Me gusta bastante } \\
\text { *Categoría: agradable }\end{array}$ & $\begin{array}{l}\text { Ecoturismo } \\
{ }^{*} \text { Senderismo interpre- } \\
\text { tativo } \\
{ }^{*} \text { Campamento } \\
\text { *Taller de educación } \\
\text { ambiental } \\
{ }^{*} \text { Caminata }\end{array}$ & \\
\hline $\begin{array}{l}\text { UP4 } \\
\text { Pastizal }\end{array}$ & $\begin{array}{l}\text { Docentes } \\
\text { * Me gusta bastante } \\
\text { *Categoría: distinguido } \\
\text { Alumnos } \\
{ }^{*} \text { Me gusta bastante } \\
{ }^{*} \text { Categoría: distinguido }\end{array}$ & $\begin{array}{l}\text { Ecoturismo } \\
{ }^{*} \text { Observación sideral } \\
{ }^{*} \text { Campamento } \\
{ }^{*} \text { T. Educación ambiental } \\
{ }^{*} \text { Cabalgata } \\
\text { Turismo rural } \\
{ }^{*} \text { Agroturismo }\end{array}$ & \\
\hline $\begin{array}{l}\text { UP5 } \\
\text { Manglar }\end{array}$ & $\begin{array}{l}\text { Docentes } \\
\text { *Me gusta bastante } \\
\text { *Categoría: fantástico } \\
\text { Alumnos } \\
\text { *Me gusta mucho } \\
\text { *Categoría: fantástico }\end{array}$ & $\begin{array}{l}\text { Ecoturismo } \\
\text { *Taller de reforestación } \\
\text { *Paseo en lancha } \\
\text { *Safari fotográfico } \\
\text { *Pesca recreativa } \\
\text { *Observación de eco- } \\
\text { sistema }\end{array}$ & \\
\hline $\begin{array}{l}\text { UP6 } \\
\text { Asentamiento } \\
\text { humano }\end{array}$ & $\begin{array}{l}\text { Docentes } \\
{ }^{*} \text { Me gusta algo } \\
\text { *Categoría: sin interés } \\
\text { Alumnos } \\
{ }^{*} \text { Me gusta poco } \\
{ }^{*} \text { Categoría: sin interés }\end{array}$ & $\begin{array}{l}\text { Ecoturismo } \\
{ }^{*} \text { Campamento } \\
\text { Turismo rural } \\
{ }^{*} \text { Agroturismo } \\
{ }^{*} \text { Gastronomía } \\
{ }^{*} \text { Medicina tradicional }\end{array}$ & A \\
\hline
\end{tabular}




\section{Conclusión}

El paisaje de la zona de estudio ha sufrido cambios significativos en su ecosistema, lo cual ha repercutido en la calidad visual de su belleza escénica. Empero, pese a que las condiciones del área han cambiado, los cinco recursos paisajísticos y las seis unidades presentadas recibieron valoraciones altas en relación con su belleza paisajística.

Por lo tanto, con base en los resultados de esta investigación, se concluye que la reserva ecológica Río Playa sí tiene potencial para realizar actividades de turismo de naturaleza, principalmente aquellas de bajo impacto en el ambiente, como el ecoturismo y el turismo rural. Si bien la reserva no contiene atractivos excepcionales, capaces de motivar por sí solos una corriente de visitantes del mercado interno de larga distancia o externo, sí cuenta con los elementos necesarios para atraer una corriente turística local. Los recursos con los que cuenta pueden complementarse con atractivos turísticos de mayor jerarquía en el municipio de Comalcalco, ya que los atractivos de la zona de estudio no implican pernoctación, a menos que se trate de actividades de campamento.

No obstante, para llevar a cabo proyectos en esta zona habrá que trabajar con la parte social, pues el desarrollo de actividades de turismo de naturaleza requiere la integración de los actores participantes. En este sentido, los resultados de las entrevistas respecto a los proyectos anteriores fueron un tanto desalentadores. De ahí la necesidad de las evaluaciones periódicas de sustentabilidad en los proyectos que se ponen en marcha sobre todo en zonas protegidas, debido a que el descuido de uno de los pilares que rigen la sustentabilidad, ya sea social, económico o ambiental, puede provocar el fracaso total del proyecto. Por otra parte, es indispensable concientizar a la población sobre el valor de los recursos a su alcance, ya que la comunidad y muchos ejidatarios desconocen su decreto oficial como reserva ecológica, de ahí que sea posible presumir que no son conscientes de su importancia.

Aunque la investigación ha cumplido con los objetivos establecidos, se sugiere la realización de estudios más profundos en el área antes de impulsar las actividades turísticas aquí propuestas y, por supuesto, la elaboración de proyectos que contemplen los tres ejes de la sustentabilidad, solo así la población podrá alcanzar un mejor nivel de vida. 


\section{Fuentes consultadas}

Aramburu, M. et al. (2005). Cartografía del paisaje de la comunidad autónoma de La Rioja. La Rioja: Gobierno de La Rioja/Departamento de Proyectos y Planificación Rural-Universidad Politécnica de Madrid (Turismo, Medio Ambiente y Política Territorial).

Cárdenas Tabares, F. (2006). Proyectos turísticos. Localización e inversión. 2a ed. México: Trillas.

Cestur-Sectur y UAM (2007). Elementos para evaluar el impacto económico, social y ambiental del turismo de naturaleza en México. México: Centro de Estudios Superiores en Turismo-Secretaría de Turismo/Universidad Autónoma Metropolitana.

Conanp-Semarnat (2007). Programa de Turismo en Áreas Protegidas 2006-2012. México: Comisión Nacional de Áreas Naturales Protegidas-Secretaría de Medio Ambiente y Recursos Naturales.

INEGI (2000). Carta topográfica de Comalcalco (E15A79), escala 1:50 000. México: Instituto Nacional de Estadística y Geografía.

(2010a). México en cifras. Información nacional por entidad federativa y municipios. Comalcalco, Tabasco [en línea]. México: Instituto Nacional de Estadística y Geografía. Disponible en: http://www3.inegi.org.mx/ sistemas/mexicocifras/default.aspx?e = 27 [2014, 3 de febrero].

------ (2010b). Mapa digital de México [en línea]. México: Instituto Nacional de Estadística y Geografía. Disponible en: http://www.inegi.org.mx/geo/ contenidos/mapadigital/ [2014, 3 de febrero].

López-Hernández, E. y A. Rodríguez Luna (2007). “Educación ambiental con agricultores para un plan de conservación de la biodiversidad, 'Los Pochitoques'”. Horizonte Sanitario, 6 (3), septiembre-diciembre, 24-33. Muñoz-Pedreros, A. (2004). "La evaluación del paisaje: una herramienta de gestión ambiental”. Revista Chilena de Historia Natural, 77 (1), 139156.

Pires, P. (2011). "Marco teórico-metodológico de los estudios del paisaje. Perspectivas de aplicación en la planificación del turismo". Estudios y Perspectivas en Turismo, 20 (3), 522-541. 
Priego Santander, A. et al. (2008). Propuesta para la generación semiautomatizada de unidades de paisajes. México: Instituto Nacional de Ecología y Cambio Climático-Secretaría de Medio Ambiente y Recursos Naturales. Profepa-Semarnat (2012). Informe anual 2011 [en línea]. México: Procuraduría Federal de Protección al Ambiente-Secretaría de Medio Ambiente y Recursos Naturales. Disponible en: http://www.profepa.gob.mx/innovaportal/file/4742/1/informe_profepa_2011.pdf [2014, 3 de febrero].

Sectur (2000). Planeación y gestión del desarrollo turístico municipal [en línea]. México: Secretaría de Turismo. Disponible en: http://www.cdi. gob.mx/ecoturismo/docs/desarrollo_turistico_municipal.pdf [2014, 5 de febrero].

Sedespa (2006). Cédula de las Áreas Naturales Protegidas de Tabasco. México: Secretaría de Desarrollo Social y Protección al Ambiente. 